Revista de Psicología Vol. 36 (1), 2018 (ISSN 0254-9247)

\title{
Revelando el significado de confiar en la jefatura en el contexto de un hospital público
}

\author{
Rodrigo Yáñez Gallardo ${ }^{1}$, Alejandro Díaz Mujica², Darío Páez Rovira ${ }^{3}$ \\ Universidad de Concepción, Chile ${ }^{1,2}$, Universidad del País Vasco, España ${ }^{3}$
}

La investigación sobre confianza interpersonal ha sido escasamente analizada desde la conceptualización de trabajadores latinoamericanos. Esta investigación se propuso comprender el significado que funcionarios de la salud dan a confiar en la jefatura directa. Se utilizó un diseño descriptivo-analítico y el enfoque fenomenológico para el análisis de la información. Se entrevistó a 65 funcionarios de un hospital público. Se obtuvieron 5 categorías, las dos principales cubren el $81 \%$ de las menciones y están asociadas a "Tener buena comunicación" y "Que apoyen cuando hay problemas". Así, emerge un modelo de confianza diádica que se construye especialmente de vínculos personalizados en que los funcionarios sienten recibir protección, relacionado probablemente a un liderazgo paternalista de la jefatura, coherente con la cultura organizacional latinoamericana.

Palabras clave: Confianza, confiabilidad, liderazgo, cultura, Latinoamérica.

\section{Revealing the meaning of trust in Leadership in the context of a public hospital}

Research on interpersonal trust has been poorly analyzed from the conceptualization of Latin American workers. This research aimed to understand the meaning that health officials attribute to trust in direct leadership. A descriptive-analytical design was used, as well as a phenomenological approach for the information analysis. 65 officials of a public hospital were interviewed. A total of 5 categories were obtained, from which the main two covered $81 \%$ of the dimensions. The two main categories are related to "having good communication" and "support when there are problems". Thus, a model of dyadic trust emerges, which is constructed especially from personalized links in which the officials perceive protection,

1 Magíster en investigación social y desarrollo. Docente del Departamento de Psicología de la Universidad de Concepción. Dirección postal: Facultad de Ciencias Sociales, Universidad de Concepción, Barrio universitario, Concepción, Chile. Contacto: ryanez@udec.cl

2 Doctor en Psicología. Docente del Departamento de Psicología de la Universidad de Concepción. Dirección postal: Facultad de Ciencias Sociales, Universidad de Concepción, Barrio universitario, Concepción, Chile. Contacto: adiazm@udec.cl

3 Doctor en Psicología. Docente del Departamento de Psicología Social y Metodología de la Universidad del País Vasco. Dirección postal: Facultad de Psicología, Universidad del País Vasco, San Sebastián, España. Contacto: dario.paez@ehu.es 
probably related to a paternalist leadership with the bosses. Findings are consistent with the Latin American organizational culture.

Key Words: Trust, trustworthiness, leadership, culture, Latin America

Revelando o significado de confiança em Liderança no contexto de um hospital público A pesquisa sobre a confiança interpessoal tem sido pouco analisada desde a conceitualização de trabalhadores latino americanos. Nesta pesquisa a proposta foi de compreender o significado que os funcionários da área da saúde dão ao confiar nos seus chefes diretos. Foi utilizado um desenho descritivo-analítico e o enfoque fenomenológico para a análise da informação. Foram entrevistados 65 funcionários de um hospital público. Foram obtidos 5 categorias, sendo que as duas principais cobrem um $81 \%$ do mencionado e está associado a "Ter boa comunicação" e "Que apoiem quando tenha problemas". Assim, surge um modelo de confiança entre os dois (funcionário-chefe e chefe-funcionário) que se constrói especialmente de vínculos personalizados em que os funcionários sentem receber proteção, relacionado provavelmente a uma liderança paternalista do chefe, coerente com a cultura organizacional da América Latina.

Palavras-chave: Confiança, confiabilidade, liderança, cultura, América Latina.

\section{Révéler la signification du leadership confiant dans le contexte d'un hôpital public}

La recherche sur la confiance interpersonnelle a été peu analysée depuis la conceptualisation des travailleurs latino-américains. Cette recherche a été proposée pour comprendre le sens que les responsables de la santé donnent à la confiance dans le leadership direct. Un plan descriptif-analytique et l'approche phénoménologique pour l'analyse de l'information ont été utilisés. 65 fonctionnaires d'un hôpital public ont été interrogés. Cinq catégories ont été obtenues, les deux principales couvrent $81 \%$ des mentions et sont associées à "Avoir une bonne communication" et "Soutenir en cas de problèmes". Ainsi, émerge un modèle de confiance dyadique qui se construit notamment sur des liens personnalisés dans lesquels les responsables se sentent protégés, probablement liés à un leadership paternaliste de la direction, cohérent avec la culture organisationnelle latino-américaine.

Mots-clés: Confiance, fiabilité, leadership, culture, Amérique latine. 
Dadas las condiciones de cambio, incertidumbre y estrés que caracteriza el mundo del trabajo en la actualidad, se considera esencial la existencia de relaciones interpersonales de confianza para favorecer el desarrollo de un trabajo cooperativo dentro de las organizaciones y el manejo de conflictos (Dietz, Gillespie \& Chao, 2010; VelezCalle, Robledo-Ardila \& Rodríguez-Ríos, 2015). Sin embargo, existe el problema de que en los lugares de trabajo de diferentes contextos, sus integrantes no comparten significados comunes sobre qué es tener confianza (Whipple, Griffis \& Daugherty, 2013). Como consecuencia, probablemente se producen inconsistencias al investigarla y se generan diferencias de criterio sobre la forma más apropiada de facilitarla.

En gran medida, hasta la actualidad las investigaciones sobre confianza interpersonal han adoptado una perspectiva neopositivista, privilegiando la metodología cuantitativa, asumiendo posturas que, generalmente, buscan una forma correcta de definirla, realizando una escasa reflexión sobre las diferencias en el tiempo y entre contextos sociales para definirla (Wright \& Ehnert, 2010). Esta escasa atención se deriva del supuesto que la medición de la confianza es universal y aplicable entre culturas, siendo las investigaciones conducidas predominantemente desde Estados Unidos, significando escasos esfuerzos por asegurar la aplicabilidad de las medidas de confianza a través de un rango amplio de contextos (McLeary \& Cruise, 2015).

Es reconocido que la investigación sobre la confianza hacia las jefaturas ha sido amplia, sin embargo, se ha realizado fundamentalmente desde las metodologías cuantitativas (Wasti, Tan \& Erdil, 2011). Este enfoque ha llevado a privilegiar su investigación a través de encuestas y experimentos de laboratorio, es decir, han predominado investigaciones de aproximaciones teóricas con limitada contextualización (Wright \& Ehnert, 2010). Wright y Ehnert (2010) señalan que la perspectiva predominantemente para abordar el tema durante el siglo 20 fue la teoría 
de la elección racional, la cual ha significado una sobresimplificación en el estudio de la confianza interpersonal, dado que ha excluido el rol de las emociones y los procesos de influencia social.

En contraposición, el presente estudio asume la perspectiva del constructivismo social, la cual sostiene que los procesos narrativos son centrales en la conceptualización. De esta forma, la confianza interpersonal fundamentalmente se crea en un contexto sociocultural donde se generan conversaciones, se transmiten historias y se escuchan experiencias en el trabajo. Para Wright y Ehnert (2010) confiar es un proceso histórico-cultural y está relacionado con compartir experiencias previas, así como también, por construir orientaciones hacia el futuro con las personas. Ahora bien, esto no niega que las representaciones se elaboran a partir de las constricciones de la realidad, que no se limitan al discurso verbal consciente y tienen características estructurantes per se (Páez \& Ayesterán, 1998).

Así, se aceptaría que el significado de las relaciones interpersonales está influido por la cultura nacional y organizacional. La confianza debe ser vista como un proceso dinámico en la vida de las personas, siendo en algunas ocasiones algo vivido intensamente $y$, otras veces, como un estado de calma y poco consciente, resaltando en algunos momentos algunos elementos y en otras oportunidades otros. La confianza es un proceso que no puede ser estudiado en un vacío contextual, necesita ser comprendido en el contexto en que está inserto (Labarca, 2014). Por tanto, un supuesto de la presente investigación es que los trabajadores poseen significados asociados a tener confianza en otro, influido por la especificidad cultural en que se dan las relaciones interpersonales.

A pesar de la gran cantidad de investigaciones sobre confianza, sorprendentemente ha existido un desarrollo limitado en la investigación acerca de cómo las personas difieren culturalmente para comprender qué constituye tener confianza (Dietz et al., 2010). También, han sido escasamente estudiadas las diferencias entre las culturas organizacionales para comprender el significado de confianza. Se estima que deberían existir importantes diferencias entre organizaciones, por ejemplo, una del área servicios como la salud debiera diferir de una 
manufacturera o militar, e incluso pudieran existir diferencias dentro de ellas. De este modo, se puede esperar que en un mismo centro de salud pudieran existir diferencias significativas entre la unidad de pediatría, urgencia u oncología. También, podrían existir diferencias entre los diferentes niveles jerárquicos y, como consecuencia, es muy factible que se generen problemas de comunicación entre jefaturas y funcionarios en el caso de que existan connotaciones diferentes para comprender como construir confianza. Así, también, los investigadores pueden asumir erradamente un significado compartido en los trabajadores encuestados, siendo que en realidad pueden comprender de diferente forma la confianza (Whipple et al., 2013).

En síntesis, existe un reconocimiento que los factores culturales ha sido sub explorados y se realiza un llamado a los investigadores a alcanzar una comprensión más profunda de la confianza, menos universalistas y más sensibles a la cultura como contexto en que se desarrollan las relaciones interpersonales (Bachmann, 2010; Dietz et al., 2010; Labarca, 2014; McLeary \& Cruise, 2015; Whipple et al., 2013). De este modo, esta investigación se propone primero, descubrir los distintos sentidos que tiene la confianza diádica entre la jefatura directa y funcionarios (as) de la salud de un hospital público. En segundo lugar, construir un modelo de confianza en la jefatura a partir de las relaciones hipotéticas entre las categorías obtenidas de las respuestas de los entrevistados. Finalmente, a partir de lo anterior, se propone reevaluar la definición de confianza organizacional propuesta por Mayer, Davis y Schoorman (1995, 2007).

\section{Definición de confianza en la jefatura}

La confianza en la jefatura se puede definir como un proceso dinámico en el tiempo en el que los trabajadores llegan a tomar decisiones que implican creer y sentir que la jefatura actuará favorablemente hacia ellos sin tener certeza al respecto, y sin existir oportunidad de conocer o controlar la manera como realmente actuará (Yáñez \& Valenzuela, 2013). Para Wasti et al. (2011) y Heyns y Rothmann (2015) un modelo 
integrativo muy influyente, considerado fundacional sobre confianza interpersonal en las organizaciones, es el desarrollado por Mayer, Davis y Schooman $(1995,2007)$. Este ha sido avalado por la revisión realizada por Colquitt, Scott, y LePine (2007) y adecuado a la situación específica de la confianza hacia la jefatura (Burke, Sims, Lazzara \& Salas, 2007). El modelo de Mayer, Davis y Schooman (2007) entre otros aspectos aporta con distinguir entre: (a) la conducta de decidir confiar en otro, (b) determinantes del contexto social y (c) dos determinantes cognitivos, la predisposición a confiar y la percepción de confiabilidad del otro. De especial interés es explicar este último.

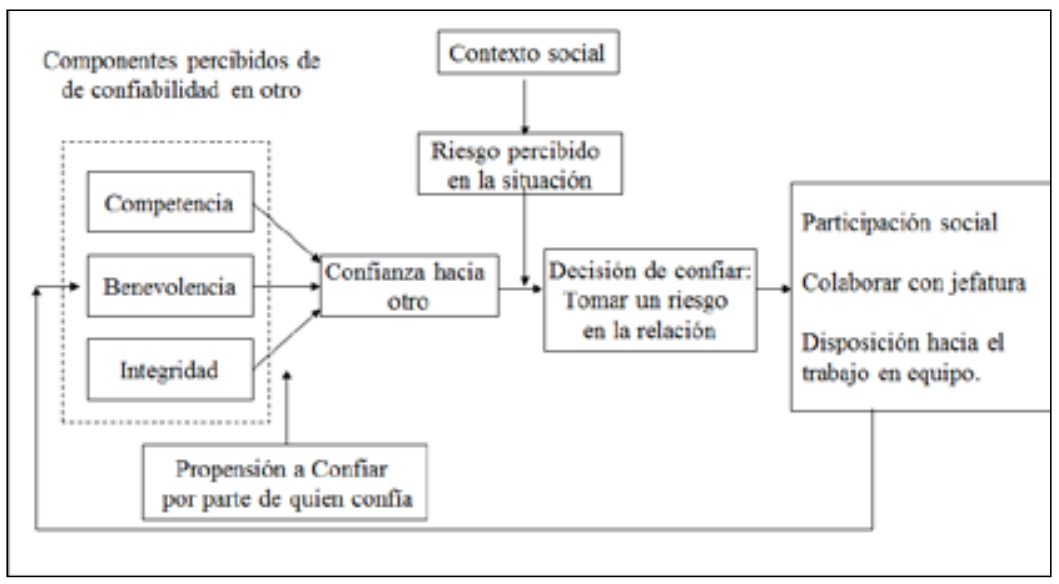

Figura 1. Modelo integrativo de confianza organizacional de Mayer, Davis y Schoorman (2007)

La confiabilidad alude a los atributos que debe poseer una persona para confiar en ella. Atributos en los que existe alto consenso serían: (a) Competencia, se define como todas aquellas cualidades necesarias para poder realizar un trabajo específico exitosamente, por ejemplo, influencia, experiencia, conocimientos y habilidades; (b) Benevolencia, se define como el cuidado e interés por el bienestar de otro sin buscar beneficios personales, corresponde al deseo de ayudar y al compromiso 
por el bienestar de los otros y simultáneamente atento a los intereses de la organización; (c) Integridad, se define como demostrar y adherir a un conjunto de principios y valores que otro juzga como aceptables, sobre todo, mostrando consistencia entre lo que sus palabras y sus acciones (Heyns \& Rothmann, 2015; Yáńez \& Valenzuela, 2013). De acuerdo con Wasti et al. (2011), las investigaciones a la fecha han confirmado la universalidad de los atributos Competencia-Integridad-Benevolencia en la formación de la confiabilidad pero se reconoce que de acuerdo al contexto puede sobresalir más una que otra. En centros de salud de nuestro medio, se ha encontrado que el desarrollo de una fuerte confianza se relaciona, especialmente, con la benevolencia de la jefatura (Yáñez \& Valenzuela, 2013).

\section{Diferencias culturales sobre confianza interpersonal en el trabajo}

Un marco seminal para estudiar las relaciones de confianza en investigaciones transculturales es el desarrollado por Hofstede, Hofstede, y Minkov (2010), quienes identificaron dimensiones que definen diferencias culturales entre sociedades y organizaciones alrededor del mundo. Doney, Cannon y Mullen (1998) propusieron que las dimensiones culturales de Hofstede influyen en la manera en que las personas desarrollan en otros. Un supuesto central es que la forma de desarrollar relaciones interpersonales de confianza en una sociedad está incluida en los valores culturales de una sociedad.

La dimensión individualismo-colectivismo implica que en el individualismo priman vínculos sociales débiles donde las personas se supone se valen por sí mismas y dan gran importancia a la consecución de objetivos personales (Hofstede et al., 2010). En las culturas individualistas en la que personas autónomas construyen relaciones voluntarias fuera de los grupos adscritos o de sangre, la confianza interpersonal generalizada es mayor. Los valores individualistas refuerzan la independencia y hacen que el sujeto dependa de sí mismo de construir relaciones voluntarias con otros, lo que exige una mayor confianza generalizada (Kramer, 1999; Yamagishi \& Yamagishi, 1994). En cambio, 
las culturas colectivistas se caracterizan por vínculos sociales estrechos, en que las personas enfatizan distinguir entre endogrupo y exogrupo, y los objetivos del endogrupo y su bienestar se valoran por encima de los del propio individuo (Hofstede et al., 2010). La desconfianza se ha asociado al colectivismo, ya que estas culturas refuerzan la cooperación en relaciones estables y específicas mientras que incrementan actitudes de desconfianza fuera del endogrupo. En estas culturas el grado de confianza general es bajo, y dada la falta de confianza de normas generales de cooperación, la incertidumbre social o desconfianza es mayor (van Hoorn, 2015).

La dimensión cultural evitación de la incertidumbre también se ha asociado a la confianza social. Las culturas con fuerte deseo de evitar la incertidumbre se caracterizan por prácticas sociales estructuradas, necesidad emocional de reglas, baja participación, estableciendo numerosas normas que incluso no se cumplen. A su vez, priman las necesidades de seguridad, estima y pertenencia, mostrando escasa disposición a aceptar riesgos y más agresivas (Hofstede et al., 2010; Kong, 2013).

Estrechamente relacionado con la confianza hacia las jefaturas está también la dimensión distancia de poder. Esta se refiere al grado en que los individuos aceptan y creen que, en las organizaciones, instituciones y sociedad, el poder esté distribuido desigualmente, como algo inevitable, legítimo o funcional (Daniels \& Greguras, 2014; Hofstede et al., 2010). La alta distancia de poder se relaciona con que los empleados respetan las jerarquías, esperan que se les diga que deben hacer y la jefatura ideal es benevolente y autocrático, es decir, un buen padre, mostrando generalmente confianza y una alta carga emocional en esta relación (Hofstede et al., 2010; Lian, Ferris \& Brown, 2012). Newman y Nollen (1996), encontraron que la participación en contextos de alta distancia de poder es visto con desconfianza y temor $y$, por tanto, las jefaturas son vistas como débiles e incompetentes (Daniels \& Greguras, 2014).

Existe un contraste muy importante para este estudio. Históricamente Latinoamérica y Chile, en particular, han sido sociedades evaluadas primando claramente las dimensiones colectivismo, fuerte evitación del riesgo y mayor distancia de poder (Hofstede et al., 2010; 
Smith, 2007). Sin embargo, la mayoría de los modelos teóricos e investigación científica que se utilizan en nuestro medio, han sido realizados en Estados Unidos, sociedad en que prevalece marcadamente las dimensiones de individualismo, débil evitación del riesgo y menor distancia de poder. Dado que las teorías del comportamiento organizacional y, específicamente, las de confianza organizacional han sido formuladas y probadas principalmente en América del Norte, se ha tendido a suponer erróneamente que los mismos resultados serán obtenidos en investigaciones realizadas en otros lugares en el mundo. Sin embargo, estudios realizados en Estados Unidos y Europa, emerge un patrón propio de cómo se desarrolla la confianza interpersonal.

Se ha encontrado que la confianza interpersonal en las culturas individualistas presenta una alta preocupación porque el otro cumpla lo acordado y menor preocupación en la calidad de las relaciones interpersonales establecidas con el otro en el trabajo (Meyer, 2014). Es decir, la preocupación por los afectos, y la relación misma, deben ser dejadas a un lado para concentrar la atención en la meta que se debe cumplir. También se afirma que se separa el dominio profesional del personal en las interacciones sociales en el trabajo, no inmiscuyéndose en el dominio de la vida personal. De este modo, corresponde que las relaciones se desarrollen solo a un nivel superficial de conocimiento personal (Wasti et al., 2011).

Branzei, Vertinsky y Camp (2007) encontraron que quienes confían en una cultura individualista colocan mayor peso en la capacidad del otro para cumplir promesas. Al respecto, se seńala que las culturas individualistas y masculinas vinculan la confianza a formas de pensar calculada, basadas en una evaluación fundamentalmente cognitiva de los costos y beneficios, y en una evaluación de la percepción de la capacidad del otro de cumplir con la tarea acordada (Ferrin \& Gillespie, 2010).

La cultura latinoamericana, al igual que la china y la árabe, al compartir una cultura colectivista y alta distancia de poder, presentan un sistema valórico en el que las relaciones interpersonales están basadas en el respeto a la autoridad, un sentido de deber y lealtad a los grupos 
(Dávila \& Elvira, 2012; Velez-Calle et al., 2015). La cultura chilena, producto de estar experimentando transformaciones socioeconómicas por la implementación del modelo neoliberal, ha ido disminuyendo el predominio de los valores colectivista, debilitándose la distancia de poder, sin embargo, habría un aumento en la evitación de la incertidumbre (Didier \& Luna, 2017). Investigaciones realizadas desde diferentes paradigmas metodológicos encuentran resultados consistentes para toda Latinoamérica, aún prevalecen los valores que enfatizan el respeto a la autoridad y al grupo (Pérez, Eades $\&$ Wilson, 2012). Sin duda, ciertos valores han ido cambiando en las personas, sin embargo, al interior de las organizaciones parece que subsisten prácticas culturales tradicionales (Didier \& Luna, 2017).

Las culturas colectivistas se caracterizan por una preocupación por la relación en el trabajo con énfasis en los vínculos afectivos y predominando las emociones positivas (Wasti et al., 2011). De esta forma, se ha encontrado que los líderes que son percibidos como efectivos en Latinoamérica presenta un estilo paternalista: una forma de control sobre los empleados a través de la imagen del padre de familia, involucrando cuidado, benevolencia y conductas de protección hacia los empleados, junto con un liderazgo autocrático, directivo, renuente a delegar y a trabajar en equipo, optando por una comunicación descendente, evitar los conflictos y que en sus relaciones son asertivos y agresivos (Castaño, de Luque, Wernsing, Ogliastri, Shemueli, Fuchs \& Robles-Flores, 2015; Dávila \& Elvira, 2012).

Cuando prima un liderazgo paternalista positivo, la disposición protectora es alta y se trata a los empleados como hijos, como resultado del supuesto tácito que ellos son incapaces de tomar decisiones propias, falta de educación apropiada o que son incapaces de tomar riesgos (Dávila \& Elvira, 2012; Castaño et al., 2015). De acuerdo con Osland, De Franco y Osland (2008) los empleados esperan que sus jefaturas se preocupen de sus vidas inclusive fuera del trabajo, fenómeno que no se encuentra en todas las culturas. Lo anterior crearía una relación simbiótica entre jefatura y empleado, generando dependencia de lealtades mutuas. De esta forma, la construcción de confianza interpersonal 
dependería de la percepción de las intenciones o motivaciones de la jefatura, de creer en su benevolencia y, su consecuencia, la conducta de cooperación de los trabajadores estaría basada en el vínculo afectivo.

La alta evitación de la incertidumbre en la cultura latinoamericana se traduce en una fuerte tendencia a buscar la seguridad, relacionado, sin duda, con el rasgo de desconfianza hacia los extraños. En el contexto laboral, se ve reflejada en enfatizar roles formales, seguimiento de reglas, respeto a las jerarquías y la búsqueda de la armonía en los grupos, es decir, no enfrentamiento de los conflictos y lealtad hacia la organización (Didier \& Luna, 2017; Páez \& Campos, 2005). A las jefaturas les cuesta delegar, optan por el contacto cara a cara e intentan construir vínculos previos a las transacciones laborales (Velez-Calle et al., 2015). De acuerdo con Páez y Campos (2005) en las sociedades colectivistas y de alto riesgo a la incertidumbre los niveles de confianza social son bajos y optan por relaciones interpersonales que provean seguridad o confianza estables y a su vez refuerzan actitudes de desconfianza y competitivas fuera del endogrupo.

En síntesis, todo lo anterior lleva a que las relaciones interpersonales sean centrales en el trabajo en Latinoamérica y, los líderes dirigen con autoridad pero afectivamente a los trabajadores, un liderazgo paternalista (Dávila \& Elvira, 2012; Pérez et al., 2012).

\section{Método}

La investigación adoptó un diseño metodológico cualitativo. Se utilizó el enfoque fenomenológico siguiendo los lineamientos de Flick (2004). Este trabajo es la continuación de una investigación sobre la construcción de la confianza y desconfianza en las jefaturas de enfermería. La investigación se realizó en un hospital público de alta complejidad, donde se realiza formación clínica e investigación universitaria, con alrededor de 2500 funcionarios. 


\section{Participantes}

Participaron 65 funcionarios de un hospital, 44 mujeres, 15 hombres y 6 que optaron por mantener anónimo, un $26.7 \%$ fueron enfermeras(os) y un $73.3 \%$ técnicos de enfermería. Se escogieron dichos profesionales porque son los que más frecuentemente son subalternos de enfermeras(os) jefes en los hospitales. Las participantes eran de 9 servicios clínicos diferentes. El método de selección fue a través de un muestreo accidental, de acuerdo a la disponibilidad de tiempo de los funcionarios en el momento en que los encuestadores se presentaron en los servicios clínicos. Se consideraron los siguientes criterios de inclusión: que los funcionarios cumplieran con ser enfermeras(os) o técnicos paramédicos; pertenecer a un servicio clínico de un hospital y tener a lo menos un año de antigüedad trabajando en dicho Servicio. De esta manera, se garantizó que los participantes tuvieran una relación de dependencia con alguna jefatura y un conocimiento de las relaciones interpersonales de confianza con las jefaturas de enfermería.

\section{Medición}

En el contexto de una entrevista semiestructurada para obtener incidentes críticos de confianza (Yáńez \& Valenzuela, 2013; Yáńez \& Cuadra, 2014), se inició la entrevista solicitándoles a los participantes que explicaran qué significaba para ellos experimentar confianza hacia sus jefaturas directa. Posteriormente, se realizaron preguntas para profundizar más en esta experiencia, dieran ejemplos y explicasen el sentido de dichas narraciones.

\section{Procedimiento}

Los entrevistadores fueron estudiantes de Enfermería y Psicología que recibieron una preparación como entrevistadores y fueron supervisados por un psicólogo. Las entrevistas se realizaron en el lugar de trabajo de los funcionarios resguardando un espacio que garantizara la privacidad para ellos. Las entrevistas tuvieron una duración aproximada de 20 minutos y fueron transcritas a versión digital. 
El proyecto de investigación fue aprobado por el Comité de Ética en Investigación de la Universidad de Concepción. Se les entregó a los participantes una carta de consentimiento libre e informado que debieron firmar previo a la entrevista. Se les garantizó el anonimato y confidencialidad de sus respuestas, además, se les solicitó un mínimo de datos personales.

\section{Análisis de datos}

Se ingresó la información al software Nvivo 10, para compilar, organizar y analizar el contenido de las entrevistas, facilitando el proceso de extracción de categorías. Una primera lectura determinó que de las 74 entrevistas, el contenido de 65 de ellas era completamente atingente a lo investigado. Se siguió una propuesta fenomenológica para el análisis de contenido de los datos. El proceso de codificación tuvo dos etapas. En la primera, a través de una codificación abierta se identificaron las unidades de significado general $y$, posteriormente, las relevantes para el significado de tener confianza en la jefatura directa. Ello implicó la lectura recurrente de las narraciones, realizando comparaciones entre ellas y el investigador se abocó a partir de un análisis comprensivo a que las categorías emergieran de los patrones comunes encontrados. Esto fue realizado por uno de los investigadores y, posteriormente, fue evaluada por el segundo investigador en forma independiente, realizándose una discusión de las diferencias encontradas hasta lograr un consenso interjueces.

En una segunda etapa, el objetivo fue agrupar las unidades que tenían un contenido compartido entre ellas, obteniéndose de este modo, categorías. Este procedimiento implicó que ambos investigadores en forma independiente construyeron agrupaciones, se discutieron las diferencias encontradas y se logró un consenso. Posteriormente, se calcularon las frecuencias de mención de cada categoría y se procedió a la elaboración de una tabla con los resultados. 


\section{Resultados}

\section{Resultados descriptivos}

Dado que algunas de las 65 entrevistas procesadas hicieron mención a más de un aspecto para explicar qué significaba confiar en las jefaturas, el total de referencias analizadas fue de 79. Se obtuvieron 5 categorías, sin embargo, tres son las principales ya que cubren el 93.71\% de las menciones (ver Tabla 1 ).

La categoría "Tener buena comunicación" fue la que obtuvo la mayor mención, un $44.3 \%$. Esta incluye 4 subcategorías, las con mayor mención aluden a "Tener comunicación fluida y empática" y "Contar cosas personales y del trabajo" y "Confidencialidad". Todas ellas están íntimamente relacionadas y enfatizan que la jefatura escuche y sea empática. Por una parte, los funcionarios indican que desean sentir la libertad de expresarse, poder ser sinceras, transparentes, pudiendo dar sus opiniones. Por otra parte, manifiestan que tienen problemas en sus hogares y que no les es posible dejarlos afuera de su trabajo y que muchas veces necesita desahogarse o sentirse comprendidos por su jefatura. Así, se transciende el plano laboral y se abordan temas personales generalmente relacionados con sus familias. Entonces, esperan que la jefatura se dé el tiempo para realmente escuchar, y que sea empática, de modo, que entiendan al trabajador. A continuación, se presentan dos ejemplos:

"Es darle a conocer cualquier cosa que esté pasando, tanto en el trabajo como en tu vida personal, porque gran parte de tu vida te la pasas dentro del hospital. Entramos a trabajar con problemas externos...de afuera, psicológicos, familiares y entras a trabajar con eso y de repente quieres desahogarte, que te entiendan y quieres dárselo a conocer a tu jefe, no para que sea un psicólogo sino para ver que no estás en las condiciones para trabajar bien porque estas con un conflicto dentro de tu cabeza." $\left(\mathrm{E} \mathrm{n}^{\circ} \mathrm{6}\right)$

"Poder acercarme a ella sin problemas, que sepa escuchar y que también se ponga en el lugar de la otra persona. Que sea accesible para mí ante cualquier problema." (E n $\left.{ }^{\circ} 13\right)$ 
Revelando el significado de confiar en la jefatura en el contexto de un hospital público / Yánez et al.

La segunda categoría con más alta mención fue "Que apoye cuando hay problemas", con un $36.7 \%$ de las menciones. En un contexto organizacional caracterizado por una alta diferenciación jerárquica y respeto a la autoridad se estaría haciendo referencia a ser protegidos por la jefatura. Por ejemplo, se hace referencia a situaciones donde existe un problema, o se ha cometido un error de procedimiento o se necesita obtener un permiso por un problema personal, entonces se espera no ser criticado, poder consultar, y recibir apoyo emocional, ánimo o consejo o, bien, una ayuda concreta como obtener información o un permiso. A continuación, se presentan dos ejemplos:

"(Si yo tengo) un paciente que tiene un problema y espero que (el jefe) no me contradiga enfrente de él (paciente), que no me rete por las cosas que hago sino que me enseñe en caso de que cometa algún tipo de error y que me vaya a escuchar por algún problema que tenga y me ayude a solucionarlo." $\left(\mathrm{E} \mathrm{n}^{\circ} 17\right)$

"Si yo tengo problemas alguna dificultad algo y voy donde mi jefa y le cuento sabe que paso tal y cuál cosa que puedo hacer, ella me va a tratar de orientar y a la vez va a ella guardar esa información no la va a repartir con el resto de los funcionarios si no que se lo va a guardar para ella y va a tratar de ver las probables soluciones a ese problema, o sea eso para mi es confiar en alguien." (E n⿳62)

La tercera categoría fue "Facilita trabajar bien" y obtiene un $12.7 \%$ de las menciones. En esta categoría se conjugan varios elementos, los funcionaros siente que son respetados y le facilitan realizar su trabajo y, así puede trabajar tranquilo en forma relajada, con deseos. Otro contenido que manifiestan los entrevistados es un interés por desarrollarse profesionalmente ya que posibilita la participación en el equipo de trabajo y se lleguen a delegan funciones en él. A continuación, se presentan dos ejemplos:

"Es como lo fundamental para tener una buena participación de todos los integrantes del equipo de salud y del turno, entonces sin confianza uno no podría desarrollarse como de forma plena en el trabajo, en las actividades que se realizan acá es como, yo no podría dudar del trabajo que hace mi compañera si estoy trabajando con ella es como lo principal para trabajar en equipo." (E n $\left.{ }^{\circ} 11\right)$ 
"Para mi significa respetarse mutuamente, entregarse conocimientos porque uno muchas veces puede ayudar a jefas que están recién ingresando y por supuestos los jefes enseñan mucho más." (E n¹9)

\section{Tabla 1}

Frecuencias y porcentajes de las categorias sobre el significado de confianza en la jefatura de enfermería

\begin{tabular}{ccc}
\hline Categoría & Frecuencia & Porcentaje \\
\hline
\end{tabular}

1. Tener buena comunicación

1.1. Tener comunicación fluida y empatía $\quad 21$

$\begin{array}{lll}\text { 1.2. Contar cosas personales y del trabajo } & 9 & 44.3 \% \\ \text { 1.3. Confidencialidad } & 4 & \\ \text { 1.4. Ser sincero } & 1\end{array}$

2. Que apoyen cuando hay problemas

2.1 Ante necesidad, recibir apoyo

$36.7 \%$

3. Facilita trabajar bien
3.1. Ayuda a hacer bien el trabajo
10
$12.7 \%$

4. La jefatura hace bien su trabajo

4.1. Cuando hace bien el trabajo

4.2. Es justo y equitativo

1

2

$5.1 \%$

4.3. Delega

1

5. Ser amigo con límites

\begin{tabular}{lcc} 
5.1. Amigo con límites & 1 & $1.3 \%$ \\
\hline Total & 79 & $100 \%$ \\
\hline
\end{tabular}

\section{Resultados analiticos relacionales}

A partir de los resultados descriptivos anteriores, se realizó un análisis relacional que generó un modelo comprensivo hipotético, basado en la vinculación que existe entre las principales categorías encontradas (ver Figura 2). 


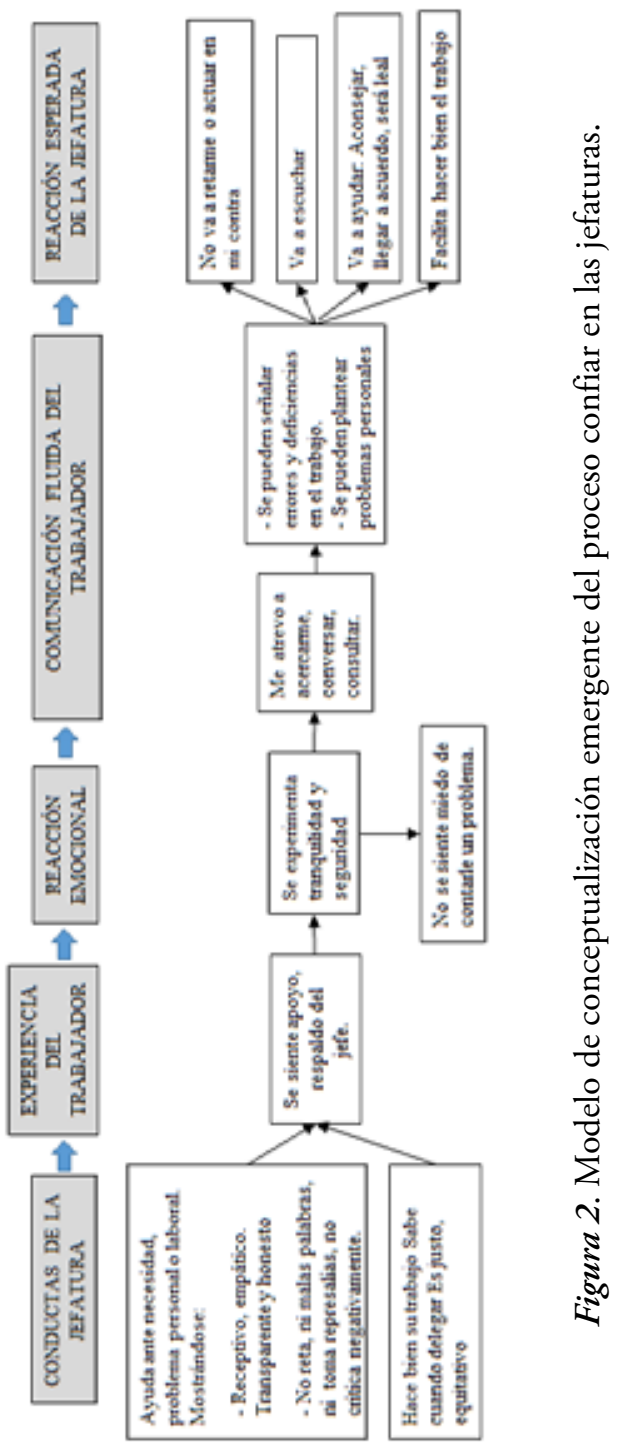


De acuerdo a los resultados, la mayoría de entrevistados asociaron la confianza en la jefatura al contexto de que un trabajador que enfrenta algún tipo de dificultad en su trabajo, quedando en una situación riesgosa y, por ende, de vulnerabilidad. La respuesta de la jefatura, que podría ser negativa, se muestra comprensiva y empática, apoyando emocionalmente o ayudando instrumentalmente al trabajador. De este modo, los trabajadores indican que dicha experiencia genera en ellos un estado emocional positivo de seguridad y tranquilidad en el trabajador, eliminando el temor hacia la jefatura. Luego, el desarrollo de esta confianza personalizada tendría el efecto que el trabajador muestra disposición a acercarse a la jefatura, lo más mencionado, es que se facilitaba la comunicación. Además, no solo el trabajador se motiva a plantearle problemas circunscritos a lo laboral, fue frecuente que manifestaran desear compartir problemas personales con la jefatura. Esta apertura del trabajador está fundada en la expectativa que al ser su jefatura confiable, los escuchará, les enseñará y ayudará en el plano personal y laboral.

\section{Discusión}

Basados en que los conceptos no tienen un significado unívoco, inclusive los de índole científico, un supuesto de esta investigación es que la conceptualización y operacionalización de confianza en la jefatura predominante no se ha comprendido y medido efectivamente, debido a que se ha considerado escasamente la diversidad cultural. Se ha planteado que es a partir de las conversaciones, de la reflexión de la propia experiencia y la cultura en la que se está inserto como los funcionarios de los hospitales toman conciencia de que quiere decir cuando hablan de confiar en las jefaturas directas. Así, este estudio busca explorar los distintos sentidos latentes sobre este término, en el contexto de un centro de salud latinoamericano y elaborar un modelo coherente con dichos significados.

Primero, se encontró que existe un alto consenso entre los funcionarios de la salud sobre el significado de tener confianza en la jefatura. 
Revelando el significado de confiar en la jefatura en el contexto de un hospital público / Yánez et al.

Si bien, se obtuvieron cinco categorías diferentes que reflejan cierta diversidad semántica al respecto, predominaron claramente dos categorías, "Tener buena comunicación” y "Que apoyen al personal cuando tienen problemas" que representan el $81 \%$ de las menciones.

De las entrevistas destaca un significado en especial: un $44.3 \%$ de los entrevistados asociaron confiar en la jefatura con la existencia de una buena comunicación. Por una parte, que la jefatura establezca un vínculo cercano, los escuche, muestre empatía y, en segundo lugar, de este modo, los funcionarios sienten la libertad de poder expresar sus preocupaciones ante la jefatura. Estas respuestas indican que los(as) funcionarios (as) asocian confiar con uno de los principales efectos que tienen las relaciones interpersonales de confianza: facilitar la comunicación entre las personas. Este resultado corroboraría el impacto importante de desarrollar confianza en las organizaciones para mejorar su eficiencia. Con respecto al contenido mismo de esta comunicación, ellos no solo aludían a poder expresar problemas vinculados al trabajo, sino también personales, lo que podría estar mostrando un cierto grado de intimidad que los funcionarios esperan establecer con su jefatura. Que la comunicación vaya más allá del plano laboral y se incluya el dominio personal, podría aportar evidencia de que la conceptualización de los funcionarios de la salud con respecto a las relaciones interpersonales con la jefatura son coherentes con los valores de las culturas colectivista planteadas por Wasti et al. (2011).

En segundo lugar, se mencionó la categoría "Que apoyen cuando hay problemas" con un $36.7 \%$ de menciones, es decir, los entrevistados asociaron confiar en la jefatura con recibir apoyo emocional y/o ayuda instrumental cuando tienen algún problema. Este resultado podría estar mostrando que la expectativa al rol de jefatura sería que, dado que en el trabajo existen problemas, se esperaría una preocupación personalizada de la jefatura por ayudarlos, podría asumirse que existe una condición necesaria para trabajar en armonía, esto es: saber que se va ser protegido por un líder benevolente.

Considerando estas dos categorías, tanto recibir apoyo como buena comunicación con la jefatura reforzaría la hipótesis del predominio de 
valores culturales colectivista en estos trabajadores de la salud. Lo anterior es coincidente con lo planteado por Wasti et al. (2011), Branzei et al. (2007), Velez-Calle et al. (2015) y Castaño et al (2015), donde los trabajadores que pertenecen a la cultura latinoamericana muestran valores coherentes con valores colectivistas. Trabajan en estructuras organizacionales jerárquicas donde establecen una relación de alta dependencia con la jefatura que confirma una alta distancia de poder y hacen sentido a los funcionarios de un líder autoritario-benevolente, esperando de él una comunicación cercana, un trato personal que atienda a sus problemas y que sea un aporte efectivo para resolverlos. Estos resultados también son coherentes con la conjetura planteada por Didier y Luna (2017), quienes plantean que los cambios en los valores en las personas y los cambios que viven las organizaciones, no se han traducido en cambios significativos en las prácticas de gestión al interior de las organizaciones.

En ciertos aspectos las personas son menos colectivistas, son más competitivas entre sí, establecen menos distancia de poder con la autoridad, sin embargo, la cultura de las organizaciones se mantendrían las formas tradiciones de las relaciones sociales de la cultura latinoamericana: se construyen relaciones de confianza en el trabajo basadas en las relaciones afectivas, en la construcción de intimidad, en la formación de grupos cohesivos, en la búsqueda de apoyo mutuo y mostrando desconfianza a quienes no son parte de su círculo cercano.

La tercera categoría obtenida "Facilita realizar el trabajo" obtuvo una baja mención, solo un $12.75 \%$. Se estima que esta categoría se relaciona estrechamente con los valores de las culturas individualistas, una preocupación por cumplir adecuadamente con las metas en el trabajo (Wasti et al., 2011). Estos resultados también podrían considerarse como evidencia de la cultura latinoamericana, el bajo porcentaje obtenido mostraría que se coloca en un segundo plano el valor individualista de cumplir con las metas comprometidas.

También los resultados obtenidos mostrarían que de las tres dimensiones de la confiabilidad de los modelos de Mayer et al. (2007), Colquitt et al. (2007) y Burke et al. (2007), la más importante en el contexto de esta investigación sería la percepción de benevolencia. 
Como mostró la investigación de Yáñez y Cuadra (2014) y la de Wasti et al. (2011) ambas utilizando incidentes críticos de confianza, lo determinante para experimentar alta confianza se logra con el desarrollo de un vínculo afectivo positivo con la jefatura acompañado de experimentar la emoción de sentirse valorado, de experimentar seguridad psicológica y gratitud, así espontáneamente la disposición a cooperar con la jefatura. Estos resultados realzan la importancia de los procesos afectivos en la relación con la jefatura, por ello, se podría hablar de una confianza personalizada y validaría la crítica al modelo de Mayer et al. (2007) en el sentido que reflejaría la cultura individualista norteamericana, la cual limita la importancia de los factores relacionales y afectivos (Wasti et al., 2011). Lo previamente seńalado no niega la relevancia de las dimensiones competencia e integridad de las jefaturas pero las colocaría en un segundo plano para desarrollar confianza.

A partir de los resultados discutidos y, en especial, del ordenamiento creado en la Figura 1, donde se presenta un modelo emergente del proceso de confiar en la jefatura al contexto de la cultura latinoamericana, se propone una nueva definición de confianza en la jefatura: Es la decisión del funcionario de correr un riesgo y establecer una comunicación fluida y honesta con su jefatura, basado en el sentimiento de seguridad que experimenta ante el apoyo emocional o ayuda instrumental brindada por la jefatura cuando enfrenta problemas en su puesto de trabajo o dificultades personales.

Algunas implicaciones prácticas se pueden extraer de los resultados obtenidos. Se concuerda con Velez-Calle et al. (2015) que se debe ser cuidadoso de importar modelos de gestión y de construcción de confianza que no son acordes a los valores donde se aplican. Una fuente grave de conflicto organizacional podría ser si las jefaturas adscriben a modelos de gestión centrados exclusivamente en el cumplimiento de la tarea, mientras los trabajadores están centrados en la relación afectiva. Siguiendo los planteamientos de Branzei et al. (2007) los empleados no responden positivamente a jefaturas que establecen relaciones superficiales, ellos optan por una mayor profundidad en su dependencia con las jefaturas. 
Se estima que los trabajadores se adecúan a las transformaciones sociales privilegiando la gestión participativa en las organizaciones pero, a su vez, coherente con el arraigo de la cultura organizacional latinoamericana. Subsiste en los trabajadores una alta distancia de poder que valora el estilo de liderazgo paternalista de mando autocrático, que ejerce control sobre los trabajadores, gradualmente dispuesto a delegar y fomentar el trabajar colaborativo y que, a su vez, construye confianza a partir de la creación de un fuerte vínculo y protección de sus empleados.

En el contexto de los centros de salud, el liderazgo en enfermería se ha descrito predominantemente autoritario y burocrático (De Lima et al., 2011; Lanzoni \& Meirelles, 2011), en climas organizacionales descritos como rígido, intolerante al conflicto y que legitima los comportamientos abusivos, como una forma habitual de relación (Castellón, 2011). En una cultura organizacional de la confianza, las actitudes de los profesionales de enfermería con roles de liderazgo deberían tomar los elementos positivos de la cultura paternalista, mostrando un interés por desarrollar una buena comunicación con los funcionarios, una orientación hacia el grupo y mostrando una preocupación holista por el bienestar de ellos e incluso, interviniendo a favor de los problemas personales de ellos.

Las principales limitaciones del presente estudio fueron: Se exploró con moderada profundidad la conceptualización de la confianza porque se realizó en el contexto de una entrevista mayor que también abordaba otros tópicos. Mayor detalle puede obtenerse de focalizar la entrevista únicamente en la conceptualización de la confianza. Se enriquecería el análisis efectuando comparaciones entre las respuestas brindadas por trabajadores de diversos servicios dentro de un hospital. Como lo han hecho ver algunos investigadores (Smith, 2007), las sociedades que integran Latinoamérica presentan importantes similitudes entre sí y, que en esta investigación se han resaltado, sin embargo, también se debe reconocer que existen diferencias, por tanto, se debe ser cauto respecto a la capacidad de generalización de los resultados de esta investigación. Como se ha mencionado en esta investigación, se valora desarrollar una 
perspectiva emic que favorezca una comprensión de los procesos de confianza en la jefatura que respete las variaciones más locales.

Dadas las escasas investigaciones sobre confianza interpersonal en el contexto organizacional latinoamericano y dada su relevancia teórica como práctica, se sugiere aumentar las investigaciones en este tópico. Se recomienda también privilegiar la investigación cualitativa para descubrir los matices existentes en los diversos contextos organizacionales. Específicamente, sería beneficioso determinar si existen diferencias en las conceptualizaciones de los funcionarios de la salud de acuerdo al género y replicar esta investigación en otros centros de salud como en otros contextos, de modo, de poder evaluar la posibilidad de generalizar los resultados obtenidos. Por último, futuras escalas de confianza interpersonal que se construyan en nuestro medio, se considera que no deben considerar únicamente los modelos teóricos extranjeros dominantes sino que, además, las conceptualizaciones arraigada en nuestra cultura.

Esta investigación buscó descubrir el significado latente del constructo confianza en el contexto de la relación con la jefatura de enfermería en un hospitalario público. Lo principal fue haber encontrado que la confiabilidad de las jefaturas depende especialmente de su benevolencia. Estos resultados podrían estar revelando tanto aspectos universales que confirman modelos ya existentes de confianza en la jefatura como específicos a la cultura latinoamericana. Específicamente, se podría asociar a rasgos colectivistas de la cultura latinoamericana donde la confianza es muy personalizada ya que está más fundada en el afecto que en lo cognitivo. De ser así, podría mantener vigencia la existencia de un estilo de liderazgo paternalista en las jefaturas en los centros de salud. 


\section{Referencias}

Bachmann, R. (2010). Towards a context sensitive approach to researching trust in inter organizational relationship. En M. Saunders (Ed.), Organizational Trust: A Cultural Perspective. (pp. 87-126). Cambridge: Cambridge University Press. https:// doi.org/10.1017/CBO9780511763106.004

Branzei, O., Vertinsky, I. \& Camp, R. D. (2007). Culture-contingent signs of trust in emergent relationships. Organizational Behavior and Human Decision Processes, 104(1), 61-82. https://doi. org/10.1016/j.obhdp.2006.11.002

Burke, C., Sims, D., Lazzara, E. \& Salas, E. (2007). Trust in leadership: A multi-level review and integration. The Leadership Quarterly, 18(6), 606-632. https://doi.org/10.1016/j.leaqua.2007.09.006

Castaño, N., de Luque, M. F. S., Wernsing, T., Ogliastri, E., Shemueli, R. G., Fuchs, R. M. \& Robles-Flores, J. A. (2015). El Jefe: Differences in expected leadership behaviors across Latin American countries. Journal of World Business, 50(3), 584-597. https://doi. org/10.1016/j.jwb.2014.12.002

Castellón, A. (2011). Violencia laboral en enfermeras: explicaciones y estrategias de afrontamiento. Revista Latino-Americana de Enfermagem, 19(1), 156-163.https://dx.doi.org/10.1590/S010411692011000100021

Colquitt, J., Scott, B. \& LePine, J. (2007). Trust, trustworthiness, and trust propensity: A metaanalytic test of their unique relationships with risk taking and job performance. Journalof Applied Psychology, 92(4), 909-927. https://doi.org/10.1037/0021-9010.92.4.909

Daniels, M. A. \& Greguras, G. J. (2014). Exploring the nature of power distance implications for micro-and macro-Level theories, processes, and outcomes. Journal of Management, 40(5), 1202-1229. https://doi.org/10.1177/0149206314527131

De Lima, L., Coelho, S., Adyles, L., Biolchi, T., Pires, D. \& Schubert, V. (2011). Influencia de los estilos de liderazgo del enfermero 
Revelando el significado de confiar en la jefatura en el contexto de un hospital público / Yánez et al.

en las relaciones interpersonales del equipo de enfermería. Enfermeria Global, 10(22), 1-9.

Dietz, G., Gillespie, N. \& Chao, G. (2010). Unravelling the complexities of trust and culture. En M. Saunders, D. Skinner, G. Dietz, N. Gillespie \& R. Lewicki (Eds.), Organizational Trust. A Cultural Perspectives Cambridge: Cambridge University Press. https://doi.org/10.1017/CBO9780511763106.002

Davila, A. \& Elvira, M. M. (2012). Humanistic leadership: Lessons from Latin America. Journal of World Business, 47(4), 548-554. https://doi.org/10.1016/j.jwb.2012.01.008

Didier, N. \& Luna, J. (2017). ¿Dónde estamos? La cultura laboral chilena desde Hofstede. Revista Colombiana de Psicología, 26(2), 295-311. https://doi.org/10.15446/rcp.v26n2.60557

Doney, P. M., Cannon, J. P. \& Mullen, M. R. (1998). Understanding the influence of national culture on the development of trust. Academy of management review, 23(3), 601-620.

Ferrin, D. \& Gillespie, N. (2010). Trust differences across nationalsocietal cultures: Much to do, or much ado about nothing. En M. Saunders (Ed.), Organizational Trust: A Cultural Perspective. Cambridge: Cambridge University Press. https://doi. org/10.1017/CBO9780511763106.003

Flick, U. (2004). Introducción a la Investigación cualitativa. Madrid: Ediciones Morata.

Heyns, M. \& Rothmann, S. (2015). Dimensionality of trust: An analysis of the relations between propensity, trustworthiness and trust. Journal of Industrial Psychology, 41(1), 01-12. https://doi. org/10.4102/sajip.v41i1.1263

Hofstede, G., Hofstede, G. J. \& Minkov, M. (2010). Cultures and organizations: Software of the mind. Londres: McGraw-Hill.

Kong, D. T. (2013). Examining a climatoeconomic contextualization of generalized social trust mediated by uncertainty avoidance. Journal of Cross-Cultural Psychology, 44(4), 574-588. https://doi.org/10.1177/0022022112466700 
Labarca, C. (2014). International business and trust. The Sino-Chilean experience. International Journal of Cross Cultural Management, 14 (3), 323-342. https://doi.org/10.1177/1470595814542258

Lanzoni, G. \& Meirelles, B. (2011). Liderazgo del enfermero: una revisión integradora de la literatura. Revista Latino-Americana de Enfermagem, 19(3), 651-658. https://doi.org/10.1590/ S0104-11692011000300026

Lian, H., Ferris, D. L. \& Brown, D. J. (2012). Does power distance exacerbate or mitigate the effects of abusive supervision? It depends on the outcome. Journal of Applied Psychology, 97(1), 107. https://doi.org/10.1037/a0024610

McLeary, C. N. \& Cruise, P. A. (2015). A context-specific model of organizational trust: An examination of cognitive and socioaffective trust determinants in unique cultural settings. Cross Cultural Management, 22(2), 297-320. https://doi.org/10.1108/ CCM-11-2013-0180

Mayer, R., Davis, J. \& Schoorman, F. (1995). An integrative model of organizational trust. Academy of Management Review, 20, 709-734.

Mayer, R., Davis, J. \& Schoorman, F. (2007). An integrative model of organizational trust. En R. Kramer (Ed.), Organizational Trust: $A$ reader (pp. 82-98). New York: Oxford University Press.

Meyer, E. (2014). The culture map: Breaking through the invisible boundaries of global business. New York: PublicAffairs.

Osland, J. S., De Franco, S. \& Osland, A. (2008). Organizational implications of Latin American Culture: lessons for the expatriate manage. Revista Economia \& Gestão, 7(14), 109-120.

Paez, D. \& Ayesterán, S. (1998). Presentación. Los desarrollos de la psicología social en España. En D. Páez y S. Etxeberria (Eds.), Los desarrollos de la psicología social en España (pp. 7-18). Madrid: Fund. Infancia y Aprendizaje.

Páez, D. \& Campos, M. (2004). Cultura, evitación de la incertidumbre y confianza interpersonal. En I. Fernández, S. Ubillos, 
E. Zubieta y D. Páez (Eds.), Psicología social, cultura y educación (pp. 537-552). Madrid: Pearson Educación.

Pérez Arrau, G., Eades, E. \& Wilson, J. (2012). Managing human resources in the Latin American context: the case of Chile. The International Journal of Human Resource Management, 23(15), 3133-3150. https://doi.org/10.1080/09585192.2011.639547

Smith, P. B. (2007). Towards studies of organizational behaviour with greater local relevance. Revista de Psicología (Lima), 25(2), 177-195.

van Hoorn, A. (2015). Individualist-Collectivist Culture and Trust RadiusAMultilevelApproach.JournalofCross-CulturalPsychology, 46(2), 269-276. https://doi.org/10.1177/0022022114551053

Velez-Calle, A., Robledo-Ardila, C. \& Rodriguez-Rios, J. D. (2015). On the influence of interpersonal relations on business practices in Latin America: A comparison with the Chinese guanxi and the Arab Wasta. Thunderbird International Business Review, 57(4), 281-293. https://doi.org/10.1002/tie.21669

Wasti, S. A., Tan, H. H. \& Erdil, S. E. (2011). Antecedents of Trust across Foci: A Comparative Study of Turkey and China. Management and Organization Review, 7(2), 279-302. https://doi. org/10.1111/j.1740-8784.2010.00186.x

Welter, R. \& Alex, N. (2012). Researching trust in different cultures. En F. G. Lyon, G. Mollering y M. Saunders (Eds.), Handbook of research methods on trust (2 ed., pp. 50-59). Nortampton: Edward Elgar Publishing.

Whipple, J. M., Griffis, S. E. \& Daugherty, P. J. (2013). Conceptualizations of Trust: Can We Trust Them? Journal of Business Logistics, 34(2), 117-130. https://doi.org/10.1111/jbl.12014

Wright, A. \& Ehnert, I. (2010). Making sense of trust across cultural contexts. En M. Saunders, D. Skinner, G. Dietz, N. Gillespie y R. Lewicki (Eds.), Organizational Trust. A Cultural Perspectives (pp. 107-126). New York: Cambridge University Press. 
Yáñez, R. \& Valenzuela, S. (2013). Conductas críticas para experimentar confianza en el liderazgo en enfermería en un hospital de alta complejidad. Revista Aquichán 13(2), 186-196.

Yáñez, R. \& Cuadra, R. (2014). Emociones emergentes ante eventos significativos de confianza con jefaturas de enfermería. Psicoperspectivas, 13(2), 165-173.

Recibido: 6 de octubre, 2016

Revisado: 18 de setiembre, 2016 Aceptado: 10 de octubre, 2017 